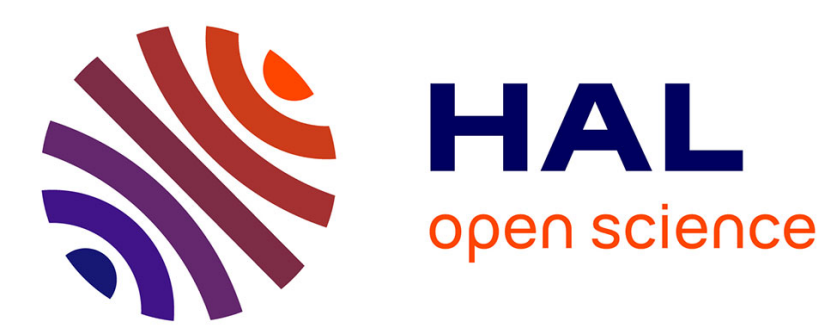

\title{
Volume of representations and birationality of peripheral holonomy
}

\author{
Antonin Guilloux
}

\section{To cite this version:}

Antonin Guilloux. Volume of representations and birationality of peripheral holonomy. Experimental Mathematics, 2018, 27 (4), 10.1080/10586458.2017.1320240 . hal-01370287

\section{HAL Id: hal-01370287 \\ https://hal.science/hal-01370287}

Submitted on 22 Sep 2016

HAL is a multi-disciplinary open access archive for the deposit and dissemination of scientific research documents, whether they are published or not. The documents may come from teaching and research institutions in France or abroad, or from public or private research centers.
L'archive ouverte pluridisciplinaire HAL, est destinée au dépôt et à la diffusion de documents scientifiques de niveau recherche, publiés ou non, émanant des établissements d'enseignement et de recherche français ou étrangers, des laboratoires publics ou privés. 


\title{
VOLUME OF REPRESENTATIONS AND BIRATIONALITY OF PERIPHERAL HOLONOMY
}

\author{
ANTONIN GUILLOUX
}

\begin{abstract}
We discuss here a generalization of a theorem by Dunfield stating that the peripheral holonomy map, from the character variety of a 3-manifold to the A-polynomial is birational. Dunfield's proof involves the rigidity of maximal volume. The volume is still an important ingredient in this paper. Unfortunately at this point no complete proof is done. Instead, a conjecture is stated about the volume function on the character variety that would imply the generalized birationality result. Some computational experimentations are described, which support the conjecture.
\end{abstract}

\section{IntRoduction}

Let $M$ be an orientable hyperbolic manifold with one cusp (e.g. a knot complement) and $\Gamma$ its fundamental group. Choose an embed$\operatorname{ding} \mathbf{Z}^{2} \rightarrow \Gamma$ of the fundamental group of the peripheral torus. Let $X(\Gamma, \operatorname{PGL}(2, \mathbf{C}))$ be the character variety:

$$
X(\Gamma, \operatorname{PGL}(2, \mathbf{C}))=\operatorname{Hom}(\Gamma, \operatorname{PGL}(2, \mathbf{C})) / / \operatorname{PGL}(2, \mathbf{C})
$$

and $X_{2}$ be the component of the hyperbolic monodromy $\rho_{\text {hyp }}: \Gamma \rightarrow$ $\operatorname{PGL}(2, \mathbf{C})$. Moreover, let $\mathrm{Hol}_{\text {periph }}$ denote, as in [13, 8], the peripheral holonomy. It is the map $X(\Gamma, \operatorname{PGL}(2, \mathbf{C})) \rightarrow X\left(\mathbf{Z}^{2}, \operatorname{PGL}(2, \mathbf{C})\right)$ naturally induced by the restriction of a representation $\rho: \Gamma \rightarrow \operatorname{PGL}(2, \mathbf{C})$ to the peripheral $\mathbf{Z}^{2}$. Note that the image of $X_{2}$ by the map Hol $_{\text {periph }}$ is the usually computed $A$-polynomial when $M$ is a knot complement. In his paper [7], Dunfield proves the following theorem:

Theorem 1.1. The map $\mathrm{Hol}_{\text {periph }}$, from $\mathrm{X}_{2}$ to its image, is a birational map.

We discuss in this paper a possible generalization of this result to the case of target group $\operatorname{PGL}(n, \mathbf{C})$ for $n \geq 2$ and multicusped $M$. The generalization for $n=2$ and $M$ multicusped is proven by KlaffTillmann [15].

Dunfield's proof uses in a crucial way the properties of the volume of representations. We will review the needed facts about this function. A major obstacle for a generalization is that the proof also uses - as does [15] - the fact that the set of points in $X_{2}$ corresponding to the hyperbolic monodromy of a Dehn surgery of $M$ is Zariski-dense in $X_{2}$. 
Rigidity of volume maximality for these points then grants the theorem. Our main problem is that this Zariski density does not hold for $n>2$.

We present in this paper a possible workaround, still using properties of the volume. Indeed we will state a conjecture, of geometric flavour, that implies the birationality in Dunfield's theorem. Hopefully, the conjecture may be easier to tackle in the general case than the birationality problem.

We will recall in section 2 some facts about the character variety and the map $\mathrm{Hol}_{\text {periph }}$ following mainly the presentation of [8]. One important result for the present paper is the local rigidity theorem (see theorem 2.1). We define the geometric representation as the composition $\rho_{\text {geom }}=r_{n} \circ \rho_{\text {hyp }}$, where $r_{n}$ is the unique irreducible representation from $\operatorname{PGL}(2, \mathbf{C})$ to $\operatorname{PGL}(n, \mathbf{C})$. The component $X_{n}$ of the character variety $X(\Gamma, \operatorname{PGL}(n, \mathbf{C}))$ containing the geometric representation is called the geometric component.

We proceed in section 3 with the definition of the volume of representations using bounded cohomology, following Bucher-Burger-Iozzi [3]. Moreover we recall another approach to this function: the more combinatorial notion of volume defined originally by Thurston for $n=2$, then by Bergeron-Falbel-Guilloux [1] for $n=3$ and fully generalized both by Dimofte-Gabella-Goncharov and Garoufalidis-Goerner-Zickert [6. 11].

Both approaches to the volume function yield valuable informations: first, through bounded cohomology one gets the volume rigidity of the geometric representation. Indeed, as proven in [3], the volume map has a unique maximum on the geometric component $X_{n}$, which corresponds to $\rho_{\text {geom. }}$. An information given by the combinatorial approach to the volume is a formula for its derivative which is only expressed in terms of the peripheral representations (see theorem 3.2). In other words, the volume map on $X_{n}$ - as in the case $n=2$ - always factors through Hol $_{\text {periph }}$ (see proposition 4.1) on a Zariski-open set.

A problem that seems rather natural is to explicitly study the volume as a function defined on $X_{n}$ and try to retrieve geometrical information from its behaviour. For example, it raises the following question, which will be relevant for this paper: if a representation has almost the maximal volume, is it almost the geometric representation? We state the following conjecture:

Conjecture 1.1. Let $M$ be an orientable cusped hyperbolic 3-manifold, $X_{n}$ the geometric component of the character variety with target group $\operatorname{PGL}(n, \mathbf{C})$ and $\mathrm{Hol}_{\text {periph }}$ the peripheral holonomy map. Then, outside of a neighbourhood of $\left[\rho_{\text {geom }}\right]$, the volume is bounded away from its maximum on $X_{n}$.

This conjecture raises an interesting and natural question per se. But it also turns out that this conjecture leads to a generalization of 
Dunfield's theorem. Indeed, we prove the following result (see section 4):

Theorem 1.2. Let $M$ be an orientable cusped hyperbolic manifold, $X_{n}$ the geometric component of the character variety with target group $\operatorname{PGL}(n, \mathbf{C})$ and $\mathrm{Hol}_{\text {periph }}$ the peripheral holonomy map. Suppose that outside of a neighborhood of $\left[\rho_{\text {geom }}\right]$, the volume is bounded away from its maximum on $X_{n}$.

Then the map $\mathrm{Hol}_{\text {periph }}$ is a birational isomorphism between $X_{n}$ and its image.

Some experimental evidences for this conjecture have been gathered and are presented in the last section 5 .

\section{Character Variety AND LOCAL Rigidity}

Most of the material of this section is already reviewed, with the same notations, in [8]. Let $n \geq 2$ be an integer. Let $N$ be a compact, oriented 3-manifold, with non-empty boundary $\partial N$, such that its interior $M$ is an oriented cusped hyperbolic 3-manifold. Denote by $\Gamma$ the fundamental group of $M$.

2.1. Character variety and geometric representation. Let $G$ be a finitely generated group. The character variety $X(G, \operatorname{PGL}(n, \mathbf{C}))$ is the GIT quotient:

$$
X(G, \operatorname{PGL}(n, \mathbf{C}))=\operatorname{Hom}(G, \operatorname{PGL}(n, \mathbf{C})) / / \operatorname{PGL}(n, \mathbf{C}) .
$$

We refer to Sikora's paper [20] for a general exposition of this object. We will restrict to two cases: first when $G=\Gamma=\pi_{1}(M)$ and second when $G=\mathbf{Z}^{2}$. We know in this setting that the character variety is an affine algebraic variety.

There is always a distinguished point in $X(\Gamma, \operatorname{PGL}(n, \mathbf{C}))$ : the class $\left[\rho_{\text {geom }}\right]$ of the geometric representation. This representation is defined as the composition of the hyperbolic monodromy $\rho_{\text {hyp }}: \Gamma \rightarrow \operatorname{PGL}(2, \mathbf{C})$ of $M$ with the (unique) irreducible representation $r_{n}: \operatorname{PGL}(2, \mathbf{C}) \rightarrow$ $\operatorname{PGL}(n, \mathbf{C})$ :

$$
\rho_{\text {geom }}=r_{n} \circ \rho_{\text {hyp }} .
$$

The character variety is not irreducible and we will not study it entirely. The main object of interest in this paper will be the geometric component $X_{n}$ : it is the unique component of $X(\Gamma, \operatorname{PGL}(n, \mathbf{C}))$ that contains $\left[\rho_{\text {geom }}\right]$.

\footnotetext{
${ }^{1}$ Recall for example that when $n=3$, the representation $r_{n}$ is also known as the adjoint representation.
} 
2.2. Peripheral holonomy and local rigidity. Let $t$ be the number of peripheral tor 2 of $M$. Let $\left(T_{i}\right)_{1 \leq i \leq t}$ be the collection of these tori and, for each $i, \Delta_{i} \simeq \mathbf{Z}^{2}$ be (a choice of) an injection of $\pi_{1}\left(T_{i}\right)$ inside $\Gamma$.

For any representation $\rho: \Gamma \rightarrow \operatorname{PGL}(n, \mathbf{C})$, one may consider its restrictions $\rho_{\Delta_{i}}$ to the various subgroups $\Delta_{i}$. This restriction defines a natural algebraic map, called peripheral holonomy:

$$
\mathrm{Hol}_{\text {periph }}: X(\Gamma, \operatorname{PGL}(n, \mathbf{C})) \rightarrow \prod_{i=1}^{t} X\left(\Delta_{i}, \operatorname{PGL}(n, \mathbf{C})\right) .
$$

This map is, together with the volume, a main character of this paper. Dunfield's theorem, indeed, states that (in the case $n=2, t=1$ ) it is a birational isomorphism between $X_{n}$ and its image. This map has already been studied through different points of view [13, 16, 2]. For the scope of this paper, we need to recall the result of Menal-Ferrer and Porti [16] (see also [2, 12]):

Theorem 2.1 (Menal-Ferrer - Porti). On a neighborhood of $\left[\rho_{\text {geom }}\right]$ in $X_{n}$, the map $\mathrm{Hol}_{\text {periph }}$ is a bijection on its image.

The theorem, as proven in the references, is more precise than this statement, giving local parameter for $X_{n}$ around $\left[\rho_{\text {geom }}\right]$. We will not need the enhanced version.

As a corollary, one may note that $\mathrm{Hol}_{\text {periph }}$ is a ramified covering (it is of finite degree). We would like to prove that its degree is indeed 1.

\section{Volume through Bounded COHOMOLOGY OR COMBINATORICS}

3.1. Bounded cohomology and rigidity. Recall the important definitions and results from the work of Bucher-Burger-Iozzi [3] about the notion of volume of a representation $\rho: \Gamma \rightarrow \operatorname{PGL}(n, \mathbf{C})$. In the cited paper, the volume map - there called Borel invariant - is defined for any $[\rho]$ in the character variety. It is the evaluation on the fundamental class of $N$ in $H_{3}(N, \partial N)$ of a suitably constructed bounded cocycle on $\operatorname{PGL}(n, \mathbf{C})$ pulled back by $\rho$. We will not review here the precise definition. For the present work, the approach of the cited article gives a crucial theorem, namely the volume rigidity result: the maximal volume is only attained once on the whole character variety, at $\left[\rho_{\text {geom }}\right]$. This rigidity theorem is a key point for the present paper. Recall that $\mathrm{Vol}_{\text {hyp }}$ is the hyperbolic volume of $M$.

Theorem 3.1 (Bucher-Burger-Iozzi). The map

$$
\mathrm{Vol}: X(\Gamma, \operatorname{PGL}(n, \mathbf{C})) \rightarrow \mathbf{R}
$$

\footnotetext{
${ }^{2}$ The reader may as well assume $t=1$ and restricts to the case of a knot complement. It will not really interfere, and may simplify notations.
} 
is onto $\left[-\frac{n\left(n^{2}-1\right)}{6} \mathrm{Vol}_{\text {hyp }} ; \frac{n\left(n^{2}-1\right)}{6} \mathrm{Vol}_{\mathrm{hyp}}\right]$. Moreover, for any point $[\rho]$ in $X(\Gamma, \operatorname{PGL}(n, \mathbf{C}))$, we have

- $\operatorname{Vol}([\rho])=\frac{n\left(n^{2}-1\right)}{6} \operatorname{Vol}_{\text {hyp }}$ iff $[\rho]=\left[\rho_{\text {geom }}\right]$,

- $\operatorname{Vol}([\rho])=-\frac{n\left(n^{2}-1\right)}{6} \operatorname{Vol}_{\text {hyp }}$ iff $[\bar{\rho}]=\left[\rho_{\text {geom }}\right]$.

The stated conjecture 1.1 comes from a question arisen during the work here presented: is it possible to "perturbate" the previous theorem. In other terms, does it holds that a representation has almost the hyperbolic volume if and only if it is almost the geometric one. Some experimentations to check this conjecture in the case $n=2$ are presented in the last section 5 ,

3.2. Combinatorics and computation of the derivative. Another approach for the volume function (and historically the first one for $n \geq 3$ ) was proposed by Thurston first, then in [1] in the case $n=$ 3 and generalized for any $n$ in [11, 6]. It is combinatorial and goes through a triangulation of $M$. The idea is to work with representations decorated by flags. For a decorated representation, each tetrahedron of the triangulation becomes a tetrahedron of flags (a hyperbolic ideal tetrahedron in the case $n=2$ ). For these tetrahedra, a notion of volume is defined by sums of Bloch-Wigner dilogarithms of cross-ratios.

We will not describe more precisely this approach. Still, two valuable informations are:

(1) The combinatorial notion of volume (defined on a Zariski-open subset of the character variety) coincide with the map Vol defined in the previous section, as explained in [3, Section 2].

(2) As a consequence, the map Vol is real analytic on a Zariski open subset of $X(\Gamma, \operatorname{PGL}(n, \mathbf{C}))$ and we know a formula for its first derivative.

Indeed, an important achievement of the combinatorial approach is that it yields a formula for the derivative of the volume. And the crucial point for us is that this formula only depends on the peripheral holonomy. Indeed, the following theorem is proven by Neumann-Zagier [17] for $n=2$, in [1, Section 11.1] for $n=3$ and its generalization to any $n$ is discussed in [6, Section 4.4].

Theorem 3.2. There is a 1-form wp on $\prod_{i=1}^{t} X\left(\Delta_{i}, \operatorname{PGL}(n, \mathbf{C})\right)$ such that $d \mathrm{Vol}$ is the pullback by $\mathrm{Hol}_{\text {periph }}$ of wp on a Zariski-open subset of $X_{n}$.

3.3. A particular case. The conjecture and the generalization of Dunfield's Theorem may be proven for the simplest example: the figure eight knot complement. We sketch the proof here.

Proposition 3.1. Let $M_{8}$ be the figure eight knot complement. Then the peripheral holonomy map, from $X_{n}$ to its image in $X\left(\mathbf{Z}^{2}, \operatorname{PGL}(n, \mathbf{C})\right)$, is a birationnal isomorphism. 
Proof. We use Theorem 1.2, which will be proven in the next section. So we have to prove that Conjecture 1.1 holds for $M_{8}$.

The ideas needed to prove that the volume is bounded away from its maximum $\frac{n\left(n^{2}-1\right)}{6} \mathrm{Vol}_{\text {hyp }}\left(M_{8}\right)$ outside of a neighborhood of $\left[\rho_{\text {geom }}\right]$ essentially go back to Thurston [21]. Indeed, as explained e.g. in [1, 8, 11], a Zariski open subset of $X_{n}$ is obtained by the monodromy of the gluing of two tetrahedra of flags. The point $\left[\rho_{\text {geom }}\right]$ corresponds to the gluing of two regular tetrahedra (as explained in [21] for the case $n=2$ ). The volume of a representation obtained by such a gluing is the sum of the volumes of both the tetrahedra [12]. The last remark is that the regular tetrahedra are the tetrahedra of maximal volume [3].

Then, choose $\varepsilon>0$ small enough, and define a neighborhood of $\left[\rho_{\text {geom }}\right]$ as the set of points in $X_{n}$ constructed as the gluing of two tetrahedra of volume at least the volume of a regular tetrahedron minus $\varepsilon$. Then, the volume of a point in $X_{n}$ which does not belong to this neighborhood, is at most $\frac{n\left(n^{2}-1\right)}{6} \operatorname{Vol}_{\text {hyp }}\left(M_{8}\right)-\varepsilon$.

This proof indeed works for any manifold that can be triangulated by few tetrahedra with respect to its hyperbolic volume, as the sister manifold of $M_{8}$ or more generally tetrahedral hyperbolic manifolds as in [10].

\section{The BIRATionality Result: A CONDitional PROOF}

We prove in this section theorem 1.2, Let $M$ be, as before, an oriented cusped hyperbolic manifold, $t$ the number of its cusps. Recall that $X_{n}$ is the geometric component of its character variety for $\operatorname{PGL}(n, \mathbf{C})$.

Throughout this section, we assume the conjecture 1.1 holds for this particular $M$ : outside a neighborhood of $\left[\rho_{\text {geom }}\right]$ in $X_{n}$, the volume function $\mathrm{Vol}$ is bounded away from its maximum on $X_{n}$.

Under this assumption, we prove theorem 1.2 stating that $\mathrm{Hol}_{\text {periph }}$ is a birational isomorphism between $X_{n}$ and its image. The first step of the proof is already proven - and crucial - when $n=2$ in [7] and [15]:

Proposition 4.1. There is a real-analytic map $V$ from a Zariski-open subset of $\operatorname{Hol}_{\text {periph }}\left(X_{n}\right)$ in $\prod_{i=1}^{t} X\left(\Delta_{i}, \operatorname{PGL}(n, \mathbf{C})\right)$ to $\mathbf{R}$ such that for any $[\rho]$ in a Zariski-open subset of $X_{n}, \operatorname{Vol}([\rho])=V\left(\operatorname{Hol}_{\text {periph }}([\rho])\right)$.

Proof. The point is to prove that wp is exact on a Zariski-open subset of the image of $X_{n}$. $V$ is then one primitive.

Consider a loop $l$ in $\operatorname{Hol}_{\text {periph }}\left(X_{n}\right)$ and assume it avoids the ramification locus of $\mathrm{Hol}_{\text {periph. }}$. Let $\bar{l}$ be a lift in $X_{n}$. The two ends of $\bar{l}$ have a volume differing by the integral $\int_{l}$ wp. If $\bar{l}$ is not a loop, we may continue the lifting of $l$ to construct a sequence of points in $X_{n}$ such that 
two consecutive points always have volumes differing by this integral. As the volume is bounded on $X_{n}$, this forces the integral to vanish.

Hence wp is exact on a Zariski-open subset of the image of $X_{n}$ and the function $V$ is its primitive whose value at $\operatorname{Hol}_{\text {periph }}\left(\left[\rho_{\text {geom }}\right]\right)$ is $\frac{n\left(n^{2}-1\right)}{6} \mathrm{Vol}_{\text {hyp }}$.

Let us proceed with the proof of theorem 2 .

Proof. Let $U$ be a neighborhood of $\left[\rho_{\text {geom }}\right]$ in $X_{n}$ such that:

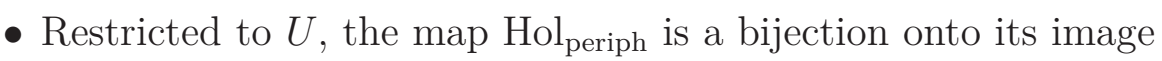
(local rigidity, see thm 2.1).

- $\operatorname{Vol}^{-1}(\operatorname{Vol}(U))=U$.

The fact that $U$ exists is a consequence of the conjecture: $\operatorname{Vol}(U)$ is a small neighbourhood of $\frac{n\left(n^{2}-1\right)}{6} \mathrm{Vol}_{\text {hyp }}$ and it has no preimage far from $\left[\rho_{\text {geom }}\right]$.

Now, let $z$ be in $\operatorname{Hol}_{\text {periph }}(U)$ such that $V$ is defined at $z$. We want to prove that $z$ has a unique preimage by $\operatorname{Hol}_{\text {periph. }}$. Let $\left[\rho_{0}\right] \in U$ be such that $z=\operatorname{Hol}_{\text {periph }}\left(\left[\rho_{0}\right]\right)$. By definition $V(z)=\operatorname{Vol}\left(\left[\rho_{0}\right]\right)$. Let $[\rho]$ be any point in $X_{n}$ such that $\operatorname{Hol}_{\text {periph }}([\rho])=z$. As $\operatorname{Vol}([\rho])=V(z)$ by the previous proposition, we get that $[\rho]$ belongs to $U$. By definition of $U$, it implies that $[\rho]=\left[\rho_{0}\right]$.

Hence there is an open subset in the image $\operatorname{Hol}_{\text {periph }}\left(X_{n}\right)$ on which the preimages of points are singletons. This means that the degree of $\mathrm{Hol}_{\text {periph }}$, from $X_{n}$ to its image, is 1 .

\section{EXPERIMENTAL EVIDENCES}

We have implemented, using Sage 7.1 [5] and Snappy [4], a threefold test to explore the validity of the conjecture in the case $n=2$. Snappy contains a census of 61911 cusped oriented manifold build as the gluing of at most 9 tetrahedra. We focus on the 1263 manifolds with at most 6 tetrahedra.

Here is a description of the test built from three different tests of increasing complexity and power. Let $M$ be an orientable manifold and $V_{M}$ its hyperbolic volume. We assume that $M$ is ideally triangulated by $\nu$ tetrahedra. Let $v_{0} \simeq 1.015$ be the volume of the regular ideal hyperbolic tetrahedron. It is the maximal volume for an ideal hyperbolic tetrahedron.

The computation will be done at the natural level for a Snappy object: the deformation variety, defined by the famous gluing equations of Thurston (see for example [22]). We work with decorated representations, i.e. monodromies of the gluing of hyperbolic realisations for the $n$ tetrahedra. The deformation variety is seen as an affine algebraic subset in $\mathbf{C}^{\nu}$ (often written in $\mathbf{C}^{3 \nu}$ to keep tracks of the symmetries of the tetrahedra): each tetrahedron is described by the cross-ratio of its vertices. 
A crucial point is that the volume is the sum of Bloch-Wigner dilogarithm of the cross-ratios and hence extend to the compactification of the character variety in $\left(\mathbf{C P}^{1}\right)^{\nu}$, as the dilogarithm is well-defined and continuous on $\mathbf{C P}^{1}$. We can check the conjecture for $M$ by proving that, at ideals points in this compactification, the generalized volume is bounded away from $V_{M}$.

Now define $k$ to be $\nu-\left\lceil\frac{V_{M}}{v_{0}}\right\rceil+1$. The meaning of $k$ is: if we know for an (ideal) decorated representation that the volume of $k$ tetrahedra among the $\nu$ vanish, then the volume of this (ideal) representation is less than $V_{M}$ : indeed, even putting the remaining $\nu-k$ tetrahedra to the maximal volume $v_{0}$, we do not reach $V_{M}$.

\section{First test}

The first test is very crude: if $k \leq 2$ then the conjecture holds for $M$. Indeed, at an ideal point at least a tetrahedron degenerates and has volume 0 . But, one may further assume that another tetrahedron is non-positively oriented, which implies it has of volume $\leq 0$. This assumption is licit because on the subset of the deformation variety where each tetrahedron is positively oriented, the volume function is convex in suitable coordinates. Hence, at the boundary of the positively oriented part, the volume of ideal points is strictly less than the maximum $V_{M}$. We need only to check the volume of ideal points outside of this boundary.

This first test grants that the conjecture holds for 1144 among the 1263 manifolds. Moreover, as it is straightforward to compute, one may check that 25986 out of the 61911 pass the test.

\section{Second test}

For the 119 manifolds left undecided by the first test, we may use the logarithmic limit set to determine the minimal number $l$ of tetrahedra degenerating at an ideal point. The reader may find in [22] a presentation of the logarithmic limit set for the deformation variety. Recall that any point in the logarithmic limit set corresponds to ideal points for the deformation variety.

Snappy may be used to recover the gluing equations defining the deformation variety. Then SageMath, through the software Gfan [14], is able to explicitly compute the points in the logarithmic limit set. Remark that any tetrahedron whose coordinate in the logarithmic limit set does not vanish does indeed degenerate at the corresponding ideal points. Thus, we compute the minimum $l$ of degenerating tetrahedra at an ideal point. If $l \geq k$, then the manifold pass the test.

It is a crude test, as each non degenerating tetrahedron is set to the maximal volume. Still further 47 manifolds pass the test. Note that this test, as written, may not be executed for manifolds with more tetrahedra. Indeed for more than 7 tetrahedra, Gfan does not compute 
the logarithmic limit. The system defining the deformation variety seems too big ( $\geq 21$ variables and equations).

The remaining 72 manifolds all have 6 tetrahedra, and have all a single cusp. We try on them a third test, more involved computationally.

\section{Third test}

We now try to compute explicitly the ideal points in $\left(\mathbf{C P}^{1}\right)^{\nu}$. This problem is hard in general (recall that we have a system with 18 variables, 18 equations and of degree around 10). A partly hand-driven computation is still often possible.

We compute Gröbner basis (with the Giac Gröbner engine [18] which appears to be the most effective for this problem), for the ideal defining the deformation variety and then the ideal $I$ defining its ideal points in $\left(\mathbf{C P}^{1}\right)^{\nu}$. This part of the computation is done in a spirit similar to [8] (trying to project on few variables and then reconstructing the whole ideal).

When $I$ is 0 -dimensional, we may then use the rational univariate representation [19], as for example in [9] through a call to the relevant Maple function to get a parametrization of ideal points. We are able to compute compute explicitly an approximated volume at ideal points by computing dilogarithms.

This procedure works for 29 from the 72 manifolds. And in each successful case we check that the maximal volume for ideal points is indeed less than the hyperbolic volume $V_{M}$. In fact, the volumes computed never exceed $10^{-13}$.

There are two risks of failure for this procedure: it happens that the ideal $I$ is not 0 -dimensional. It also happens that the computation is too long. In the first case of failure, some further study has been done on one example, enabling to check the conjecture for this example. But such a study is not at all automatic.

At the end of this threefold test, 1221 out of 1263 pass the test, giving hope for the conjecture. For the remaining 42 ones and manifolds with more than 7 tetrahedra, the computations as presented here are too complicated.

\section{REFERENCES}

1. Nicolas Bergeron, Elisha Falbel, and Antonin Guilloux, Tetrahedra of flags, volume and homology of SL(3), Geom. Topol. 18 (2014), no. 4, 1911-1971.

2. Nicolas Bergeron, Elisha Falbel, Antonin Guilloux, Pierre-Vincent Koseleff, and Fabrice Rouillier, Local rigidity for $\operatorname{PGL}(3, \mathbf{C})$-representations of 3-manifold groups, Exp. Math. 22 (2013), no. 4, 410-420. MR 3171102

3. Michelle Bucher, Marc Burger, and Alessandra Iozzi, Rigidity of representations of hyperbolic lattices in PSL(2,C) into PSL (n,C) , (2014), 1-40.

4. Marc Culler, Nathan M. Dunfield, and Jeffrey R. Weeks, SnapPy, a computer program for studying the topology of 3-manifolds, Available at http://snappy.computop.org. 
5. The Sage Developers, Sagemath, the Sage Mathematics Software System (Version 7.1), 2016.

6. Tudor Dimofte, Maxime Gabella, and Alexander B Goncharov, Kdecompositions and 3d gauge theories, arXiv preprint arXiv:1301.0192 (2013).

7. Nathan M. Dunfield, Cyclic surgery, degrees of maps of character curves, and volume rigidity for hyperbolic manifolds, Invent. Math. 136 (1999), no. 3, 623657. MR 1695208

8. E. Falbel, A. Guilloux, P.-V. Koseleff, F. Rouillier, and M. Thistlethwaite, Character varieties for $\mathrm{SL}(\mathbf{3}, \mathbb{C})$ : the figure eight knot, Exp. Math. 25 (2016), no. 2, 219-235.

9. E. Falbel, P.-V. Koseleff, and F. Rouillier, Representations of fundamental groups of 3-manifolds into $\mathrm{PGL}(3, \mathbb{C})$ : exact computations in low complexity, Geom. Dedicata 177 (2015), 229-255.

10. Evgeny Fominykh, Stavros Garoufalidis, Matthias Goerner, Vladimir Tarkaev, and Andrei Vesnin, A Census of Tetrahedral Hyperbolic Manifolds, Exp. Math. 25 (2016), no. 4, 466-481.

11. Stavros Garoufalidis, Matthias Goerner, and Christian K. Zickert, Gluing equations for $\mathrm{PGL}(n, \mathbb{C})$-representations of 3-manifolds, Algebr. Geom. Topol. 15 (2015), no. 1, 565-622.

12. Antonin Guilloux, Representations of 3-manifold groups in $\operatorname{PGL}(n, \mathbf{C})$ and their restriction to the boundary, arXiv preprint arXiv:1310.2907 (2013).

13. _ Deformation of hyperbolic manifolds in $\operatorname{PGL}(n, \mathbf{C})$ and discreteness of the peripheral representations, Proc. Amer. Math. Soc. 143 (2015), no. 5, 2215-2226.

14. Anders N. Jensen, Gfan, a software system for Gröbner fans and tropical varieties, Available at http://home.imf .au.dk/jensen/software/gfan/gfan.html.

15. B. Klaff and S. Tillmann, A birationality result for character varieties, ArXiv e-prints (2013).

16. Pere Menal-Ferrer and Joan Porti, Local coordinates for $\operatorname{SL}(n, \mathbf{C})$-character varieties of finite-volume hyperbolic 3-manifolds, Ann. Math. Blaise Pascal 19 (2012), no. 1, 107-122.

17. Walter D. Neumann and Don Zagier, Volumes of hyperbolic three-manifolds, Topology 24 (1985), no. 3, 307-332. MR 815482 (87j:57008)

18. Bernard Parisse and Renée De Graeve, Giac/Xcas, a free computer algebra system, Available at http://www-fourier.ujf-grenoble.fr/ parisse/giac_fr.html.

19. Fabrice Rouillier, Solving zero-dimensional systems through the rational univariate representation, Appl. Algebra Engrg. Comm. Comput. 9 (1999), no. 5, 433-461.

20. A. S. Sikora, Character varieties, Trans. Amer. Math. Soc. 364 (2012), no. 10, 5173-5208.

21. William P. Thurston, Three-dimensional geometry and topology. Vol. 1, Princeton Mathematical Series, vol. 35, Princeton University Press, Princeton, NJ, 1997, Edited by Silvio Levy.

22. Stephan Tillmann, Degenerations of ideal hyperbolic triangulations, Math. Z. 272 (2012), no. 3-4, 793-823.

IMJ-PRG, UPMC ANd OURAGAN, InRIA, 4, Place Jussieu, 75005 PARIS, FRANCE, ANTONIN.GUILLOUX@IMJ-PRG.FR 